\title{
e-Phaïstos
}

e-Phaïstos

Revue d'histoire des techniques / Journal of the history

of technology

VII-1 | 2019

Le travail humain

\section{Entretien avec François Vatin}

Une sociologie historique de la mesure entre sociologie de l'activité et histoire des sciences

A Historical Sociology of Measurement between Sociology of Activity and History of Science: Interview with François Vatin by Barthelemy Durrive and Marco

Saraceno

Barthélémy Durrive, Marco Saraceno et François Vatin

\section{OpenEdition}

Journals

Édition électronique

URL : http://journals.openedition.org/ephaistos/4518

DOI : 10.4000/ephaistos.4518

ISSN : 2552-0741

Éditeur

IHMC - Institut d'histoire moderne et contemporaine (UMR 8066)

Référence électronique

Barthélémy Durrive, Marco Saraceno et François Vatin, « Entretien avec François Vatin », e-Phaïstos [En ligne], VII-1 | 2019, mis en ligne le 15 avril 2019, consulté le 09 novembre 2019. URL : http:// journals.openedition.org/ephaistos/4518

Ce document a été généré automatiquement le 9 novembre 2019

Tous droits réservés 


\title{
Entretien avec François Vatin
}

Une sociologie historique de la mesure entre sociologie de l'activité et histoire des sciences

\author{
A Historical Sociology of Measurement between Sociology of Activity and History \\ of Science: Interview with François Vatin by Barthelemy Durrive and Marco
}

Saraceno

Barthélémy Durrive, Marco Saraceno et François Vatin

\section{NOTE DE L'AUTEUR}

François Vatin, sociologue et historien, professeur à l'université Paris Ouest Nanterre, nous a accordé en février 2018 un entretien autour des questions épistémologiques que soulève l'histoire des techniques métrologiques de l'activité organique pour les sciences humaines du travail. Bien que la revue e-Phaistos ait déjà publié une interview avec $\mathrm{F}$. Vatin, autour de ses recherches sur l'histoire de la mécanique ${ }^{1}$, nous avons souhaité, dans le cadre du dossier sur la mesure du travail, poursuivre l'échange. En nous concentrant sur le rôle qu'a joué l'histoire des mesures psychophysiologiques, bioénergétiques et biomécaniques pour ses recherches en sociologie du travail, la réflexion a pu se poursuivre autour de la question de l'efficacité technique dans l'épistémologie des sciences humaines. Il ne s'agit plus toutefois de la mesure de l'efficacité des premières machines à vapeur évoquée dans l'entretien de 2013, mais bien du corps en tant que « premier instrument de l'homme ». Nous remercions François Vatin pour sa disponibilité.

Pour ce qui concerne sa biobibliographie, nous renvoyons au numéro de décembre 2013 de la revue e-Phaïstos. Ajoutons-y la future publication, au Brésil, de la traduction de son ouvrage Travail économie et physique (1780-1830), sous un titre qui fait directement écho à ce dossier : As medidas do trabalho: física, economia e sociedad, Mercado de Letras, Campinas, 2019.

Lorsqu'on lit vos travaux, on a la sensation que la confrontation avec l'histoire des «sciences du corps en activité» (la physiologie, la bio-mécanique, la psychologie 
expérimentale ou encore les neurosciences) vous a été fondamentale pour aborder la question du travail d'un point de vue anthropologique comme activité créative. On peut s'étonner que pour saisir l'engagement créatif du corps humain, le sociologue que vous êtes ait dû faire un détour par des discours cherchant au contraire à réduire l'activité du corps à des fonctions modélisables. Dans quelle mesure ces discours vous ont-ils servi de «repoussoir épistémologique» pour mieux faire apparaître l'irréductibilité du corps en activité ? Dans quelle mesure, au contraire, l'étude du développement interne de ces disciplines a-t-elle été « épistémologiquement féconde » par rapport à votre propre manière d'aborder le corps au travail ?

Ma réflexion trouve son origine dans la configuration épistémologique de l'économie et de la sociologie du travail marxisantes françaises de la fin des années 1970. Y dominait un discours sur le taylorisme, dont la manifestation la plus nette a été le succès de la traduction de l'ouvrage d'Harry Braverman, Travail et capitalisme monopoliste $e^{2}$. Je travaillais à l'époque à Aix-en-Provence sur les industries à processus de production continu, dont le modèle productif - ce que j'ai appelé la «fluidité » contredisait cette conception du travail. Je m'étonnais donc de la réduction des questions du travail et de la production industrielle à la métrologie taylorienne. J'observais la fascination paradoxale des marxistes pour le taylorisme, dans lequel ils voyaient la réalisation pratique des théories de Marx. C'était là en contradiction avec l'enseignement de l'ergonomie (au sens large) attachée à une véritable observation du travail.

À cette époque d'ailleurs, avec Raymond Galle, nous avons présenté, dans la poursuite de notre première étude ${ }^{3}$, un projet de recherche, qui n'a malheureusement pas été retenu par le financeur sollicité, pour articuler la notion de fluidité avec une approche ergonomique du travail de surveillance-contrôle. Il y avait une connexion évidente avec les travaux menés à la même époque autour d'Yves Schwartz, que je n'avais pas encore personnellement rencontré. Je me souviens de conférences sur l'autoconfrontation avec $\mathrm{du}$ matériau filmique, mais mes souvenirs sont malheureusement nébuleux. À la même époque, à Toulouse, Gilbert de Terssac s'est orienté dans une direction similaire.

C'est ce rapport, que je jugeais paradoxal, des marxistes au taylorisme qui m'a conduit à vouloir rééditer Taylor ${ }^{4}$. Tous ceux qui parlaient de Taylor comme l'auteur qui aurait donné la clé du travail moderne ne faisaient rien pour le mettre à disposition des lecteurs. Inversement, j'ai voulu qu'il soit rendu disponible, précisément parce que je voulais en relativiser l'importance. D'où mon édition critique, où le texte de Taylor est éclairé par quatre textes le commentant qui lui sont contemporains.

Après, est venu s'ajouter, un peu par sérendipité, une nouvelle strate dans mon corpus intellectuel: ma découverte de la théorie mécanique du travail. Cette découverte a pour origine un autre développement de mon travail sur la fluidité industrielle: la réflexion sur l'économicité intrinsèque de la technique. Pour moi, toute mesure de l'efficacité technique est économique, en ce qu'elle incorpore une norme de valeur. Une telle réflexion était présente à l'époque dans les recherches sur les liens entre économie et thermodynamique ${ }^{5}$. Ce fut ma porte d'entrée. Très vite, j'ai découvert le travail des ingénieurs du début du XIX ${ }^{e}$ siècle autour de la mesure du travail mécanique. Je me suis immédiatement rendu compte que je n'avais pas besoin de faire de la haute épistémologie, puisqu'ils définissaient eux-mêmes le concept mécanique de travail en termes explicitement économiques. Cela m'a conduit à 
réviser un peu la façon dont l'histoire des sciences a relaté l'invention du concept de travail mécanique. J'ai insisté notamment sur le caractère central, dans cette histoire, du texte de 1799 , rédigé par Coulomb sur le travail des hommes ${ }^{6}$. À ce moment-là, mes différentes recherches se sont enchaînées les unes à la suite des autres. Cela correspond à l'agencement de l'ouvrage que j'ai publié à Bruxelles en 1999 : Le travail. Sciences et société la généalogie de ma propre pensée. Je suis parti du concept mécanique de travail pour arriver au débat du début du XXe siècle sur le modèle taylorien. Il y a donc eu ces deux histoires un peu différentes qui se sont combinées. Cela a été alimenté par différentes publications, comme la sollicitation d'Yves Clot pour son séminaire sur l'histoire de la psychologie ${ }^{8}$ ou celle de collègues suisses pour un ouvrage sur la physiologie du travail à l'ère industrielle ${ }^{9}$.

Une analyse physiologique comme celle d'Armand Imbert est un antidote extraordinaire à Taylor. Elle m'a conduit à cette formule-slogan dans un exposé que j'avais fait à Cerisy dans le colloque L'Homme point aveugle des sciences de l'homme : « un peu de mécanique éloigne du social, beaucoup y ramène $»^{10}$. Ce qui est intéressant chez Imbert, c'est qu'il utilise de manière extrêmement fine une métrologie biomécanique, mais que cette utilisation ne le conduit nullement à penser l'homme comme une machine. Il étudie la machine dans l'homme pour penser l'économie du geste. À la différence de Taylor, Imbert est un physiologiste qui analyse de manière très fine le système squeletto-musculaire. C'est ce qui le conduit à penser la complexité du geste et, au-delà, la complexité du travail tout court. Il montre ainsi que si on mesure énergétiquement, de façon comparative, le travail du portefaix du port de Sète, de l'ouvrier des chais et du facteur rural, c'est le facteur rural qui a la dépense énergétique la plus élevée et, le portefaix, la plus faible ${ }^{11}$. Cela montre bien que l'enjeu n'est pas la dépense énergétique globale, mais dans la nature des systèmes squeletto-musculaires mobilisés («les petits muscles se fatiguent plus vite que les gros ») et dans les conditions de leur mobilisation (rythme, etc.). Donc, Imbert nous fait sortir radicalement de la métrologie sommaire du travail qui est celle de Taylor, mais aussi de Jules Amar ${ }^{12}$. Si cette dernière est formulée dans des termes plus scientifiques, elle repose sur les mêmes unités de mesure englobantes. Imbert nous montre qu'il faut regarder les choses localement, aussi bien dans l'étude du corps humain que dans l'étude de son usage temporel. Par exemple, pour réguler le travail des enfants, des jeunes travailleurs, les règles consistant à limiter les charges ne sont pas pour lui la solution (un corps en formation peut très bien supporter des efforts assez violents; au contraire, il en a besoin pour se former corporellement) ; l'enjeu est celui de la continuité dans un tel effort.

Vous vous êtes confrontés avec la figure de Taylor. Comme vous avez contribué à le montrer, l'histoire des mesures du travail est beaucoup plus riche que le chronométrage tayloriste. Pourtant, l'imaginaire du chronomètre semble hanter toute étude qui rapproche les termes : mesure, corps et travail. II nous paraît par exemple difficile d'écrire une introduction à un dossier sur l'histoire de la mesure du corps au travail sans citer Taylor, alors que dans son œuvre, il est très peu question de corps. Qu'est ce que cela nous dit de notre imaginaire du corps au travail et de la mesure du corps au travail ?

Je remonterai plus loin. J'ai montré ${ }^{13}$ que l'origine de ce topos datait du début du XIX siècle, avec le texte de Pierre-Édouard Lémontey critiquant Smith de $1801^{14}$, d'où résulte la figure de l'« ouvrier-machine " (l'expression date de Lémotey). J'ai donc pu faire la généalogie de ce discours, puisque partant de Lemontey, on passe à un livre 
qui a eu un très grand succès au milieu du XIX ${ }^{e}$ siècle : Le peuple de Michelet ${ }^{15}$. Or, ce livre inspire Georges Friedmann en 1936 dans la Crise de progrè ${ }^{16}$. C'est paradoxal, puisque, contrairement à ce qu'on croit souvent, la Crise du progrès n'est pas un livre de critique de la notion de "progrès "; c'est, au contraire, une critique des auteurs critiques du progrès des années 1930. Sauf qu'en même temps, Friedmann est fasciné par le lyrisme de Michelet dans sa description romantique du «machinisme industriel » (expression qu'invente Michelet et que l'on a, a tort, attribuée à Marx, parce que le premier traducteur de Marx en français a repris le terme de Michelet pour traduire Maschinerie, soit machinery au sens d'Andrew Ure ${ }^{17}$ ). Ainsi, quand Friedmann bascule, après la Seconde Guerre mondiale, dans la critique du taylorisme, il le fait dans les termes de Michelet ${ }^{18}$. Autrement dit, une critique romantique contre-révolutionnaire du progrès (c'est bien ainsi que Lémontey situait son propre texte) a, paradoxalement, pris les habits du marxisme. Par ce biais - et notamment via Friedmann qui adopte cette topique au moment même où il rompt avec le marxisme -, on a hérité d'une lecture romantique erronée de l'œuvre de Marx.

Taylor est donc un chaînon dans une histoire remarquablement stable pendant deux siècles: le topos sur l'ouvrier-machine ne change pas de 1800 à aujourd'hui, alors même que les techniques industrielles et le travail qu'il est censé résumer n'ont cessé de changer. Ce discours de réduction de l'homme à la machine n'a pas été tellement le fait des techniciens et des ingénieurs ; il a surtout été porté par les critiques humanistes du travail moderne, qui ont saisi avec délectation les quelques auteurs qui leur offraient cette caricature, comme Taylor ou comme Amar, alors que l'on a ignoré, par exemple, Imbert. On retrouve ce que je disais précédemment : Taylor a été approprié par ceux qui entendaient s'y opposer et qui lui ont ainsi donné plus d'importance qu'il n'en mérite. C'est ça, le caractère paradoxal du discours sur le taylorisme qui est, pour une large part, un discours français. C'est une chose qui est tout à fait frappante quand on regarde dans l'ouvrage de Donald Roy, sa fameuse analyse du freinage ${ }^{19}$ : c'est exactement celle de Taylor (c'est d'ailleurs la dimension la plus intéressante de la théorie de Taylor), mais il l'ignore. Ainsi, un sociologue américain du travail des années 1950 n'avait pas lu Taylor ! Vingt ans plus tard, Harry Braverman est en fait inspiré par Friedmann, qui a été traduit! Ainsi, même dans la tradition américaine, c'est le discours français sur le taylorisme qui s'est diffusé. En citant Braverman, les Français reprenaient aux Américains ce qu'ils leur avaient donné.

La théorie de Taylor n'a donc pas servi aux ingénieurs pour définir leurs métrologies. C'est ce que j'ai montré dans mes travaux sur les débats autour du taylorisme. À l'époque même de Taylor, on trouve des critiques gestionnaires de sa pensée parfaitement judicieuses. Par exemple, dans une entreprise qui utilise des matières premières précieuses, le souci majeur du point de vue de la bonne gestion, n'est pas d'économiser le temps de travail humain, mais bien la matière première. L'obsession de Taylor d'être porté sur ce seul facteur est régulièrement dénoncée. On trouve par exemple une critique très puissante chez Émile Belot ${ }^{20}$. La devise de Taylor était « juste paye, juste tâche ». Une fois que l'on aurait conduit les ouvriers à fournir une «loyale journée de travail», il faudrait augmenter en conséquence leurs salaires. Mais Taylor remarque qu'au lieu d'augmenter le salaire journalier, on pourrait baisser la durée journalière du travail, ce qui serait selon lui préférable, puisque la productivité baisse dans les dernières heures de travail. Belot fait remarquer que, 
dans ce raisonnement, Taylor confond temps de travail et temps de production, c'està-dire qu'il ne prend pas en compte l'immobilisation des machines (le " travail mort " pour parler comme Marx). Si on réduit la durée de travail, on réduit aussi la durée d'utilisation des équipements, sauf à mettre en place un système d'équipes successives. C'est une critique gestionnaire, en rien " humaniste». Mais elle montre l'étroitesse du point de vue de Taylor : obsédé par le temps de travail direct, il n'était pas capable de concevoir ce genre de question. Sa norme d'efficacité n'a donc pas la généralité qu'on lui a souvent attribuée, du point de vue même des gestionnaires d'usines.

Je ne veux pas dire - encore une fois indépendamment de quelconque considération humaniste -, que la chasse aux « temps morts » n'a jamais sa raison d'être. C'est une des dimensions de l'efficacité industrielle et je ne cherche pas à le nier. Je dis que ça n'a pas la généralité qu'on lui attribue souvent. Le problème est notamment celui de l'arbitrage quantité-qualité. Augmenter le rythme, ça n'a pas de sens que si l'on garantit que c'est à qualité constante. Mais, supposer une "qualité constante », cela signifie introduire une autre dimension du travail, irréductible à la métrique temporelle initiale. Ramener le travail à sa mesure temporelle de type taylorienne, c'est faire comme si la question du contenu de l'activité ne se posait pas. On retrouve la figure de l'ouvrier-machine, dont le geste serait si totalement codifié, qu'il n'y aurait plus aucune interrogation sur sa pertinence, sur sa qualité. Mais si le travail est effectivement de cette nature, il vaudra toujours mieux le remplacer par un dispositif mécanique. Même dans des travaux apparemment "simples", si on fait appel à un opérateur humain, c'est parce que le geste n'est pas de cette nature, qu'on attend de lui une souplesse psychomotrice et/ou cognitive, une intelligence du geste et de l'action. C'est cette dimension-là qui ne peut pas être contenue dans la métrique tayloriste. La métrique tayloriste ne fonctionnera que dans la mesure où on considérera que cette dimension de l'activité est spontanément réglée.

L'appel à une sociologie de l'activité de travail, c'est une réponse à l'autre face du même réductionnisme temporel. Celui qui repose, non sur la métrologie mécanique, mais sur la métrologie socio-économique standard, celle du «temps de travail» prévu dans le contrat de travail. On confond ici travail et salariat. C'est clairement en opposition à cette approche, développée notamment par Dominique Méda ${ }^{21}$, dans la tradition de Hannah Arendt ${ }^{22}$, que j'ai essayé de reprendra la question de l'activité. Dans cette métrologie socio-économique, on considère par hypothèse le travail comme une " peine ", sans que pour autant on ne s'attache à mesurer cette peine par une métrologie physique. Il suffit de définir cette peine par le marché (si on paye le travailleur, c'est pour compenser la «désutilité » du travail) et par la contrainte sociale. In fine le salariat suffit à définir le caractère pénible du travail. À partir de là, on retrouve la même métrologie temporelle, à une échelle plus macro. Le travail ne serait que le temps compté comme tel par le droit de travail, dans une ignorance totale de ce qui est effectivement fait ou pas dans ce temps et dans ce qui est fait dans d'autres temps. En confondant le travail et le salariat, on ne peut plus comprendre ni l'un ni l'autre. A contrario, ma définition du travail comme "activité à vocation productive » vise à réinterroger la multiplicité des valeurs du travail.

Dans vos travaux, le rapport entre les notions de « valeur » et de «travail » est central. Vous avez notamment insisté sur la nécessité de poser cette question sans la réduire aux débats sur la valeur-travail des économistes qui renvoie l'interrogation concernant «ce qui vaut 
dans le travail ? » à celles des coûts salariaux. Or, par cette approche, vous ne cherchez nullement à évacuer la question du rôle du travail dans la production marchande, mais vous souhaitez montrer son encastrement dans une "confrontation » avec des valeurs autres que ceux du marché (éthiques, esthétiques, politiques, mais aussi médicales, environnementales, techniques... etc.). Le corps du sujet engagé dans le travail se trouve justement au cœur de cette tension entre valeurs complémentaires qu'il doit trancher pour concrétiser l'activité. Comment penser les termes de cette confrontation entre des valeurs qui semblent se légitimer sur des échelles d'évaluations incommensurables? Dans ce contexte, dans lequel le sujet est appelé à incorporer dans son activité des jugements de valeur justement incommensurables, quel rôle jouent les mesures «objectives » des sciences du corps en activité ?

Je n'essaie pas forcément de raisonner dans ces termes de commensurabilité. Je raisonne à partir d'un point de départ très pratique qui est celui de l'expérience ordinaire. Qu'est-ce que je veux dire en définissant le travail comme activité à vocation productive ? Je renvoie simplement à une définition qu'on trouve chez Marx, dans la célèbre distinction de l'abeille et de l'architecte, ou qu'on trouve dans la psychologie d'Henri Wallon ${ }^{23}$ qui définit le travail comme "activité contrainte». C'est l'opposition entre travail et jeux, au sens où le jeu se justifie par lui-même, dans l'instant. Ce qui est propre au travail, c'est qu'il est orienté par sa fin. C'est ce que j'essaie de penser dans l'idée d'une « vocation productive » : il y a du travail dès lors que l'on cherche à obtenir quelque chose et que l'activité est jugée au regard de la fin qu'on vise; ce qui permet par ailleurs de comprendre comment le jeu peut se transformer en travail. C'est cette tension entre l'activité et sa finalité (la «production ») qui va être au cœur de la réflexion. Du coup, cela pose la question de la nature même de la finalité et une multiplicité de régimes de valeur et d'instances de valorisation peuvent apparaître. Par exemple, l'activité peut être jugée au sens de la norme salariale, par rapport à ce qui est dit dans le contrat de travail, mais celui-ci est toujours «incomplet». Cette norme salariale est généralement associée à une hiérarchie professionnelle (c'est le caractère «subordonné» de la relation de travail) : cela induit la validité du jugement du supérieur hiérarchique. Mais on a aussi souvent affaire à un jugement professionnel par les pairs, à une norme, qui n'est pas salariale ou hiérarchique de ce qui constitue un travail «bien fait ». Dans certains cas (relation de service), on est aussi jugé par le client, le consommateur final ; enfin, on se juge soi-même aussi, par la façon dont on se projette dans l'activité. C'est cette multiplicité des jugements qui me paraît comme devant être prise en considération.

Cela ramène aussi, d'ailleurs, à des questions classiques de sociologie des organisations, par exemple avec l'analyse classique de Crozier sur la confrontation entre le service de fabrication qui veut à tout prix sortir les pièces parce qu'il y a des commandes à livrer et le service d'entretien qui veut ménager les machines ${ }^{24}$. Dans une opposition comme celle-là, on n'est pas dans le modèle de la confrontation salariale, ce n'est pas la lutte des classes ; les deux acteurs raisonnent du point de vue de ce qui leur paraît être l'intérêt de l'entreprise, mais l'intérêt de l'entreprise, tel qu'eux-mêmes l'incorporent à travers leurs responsabilités professionnelles. Il n'y a pas de solutions simples (qui aurait raison ou tort), mais bien des arbitrages qui sont faits in fine. C'est à travers ces arbitrages que se construit la commensurabilité. La mesure peut éventuellement servir d'aide à l'arbitrage, mais pas dans le sens où elle serait "neutre ", dénuée de valeur. Un des éléments de l'arbitrage est la question temporelle: on voit par exemple, dans le cas évoqué plus haut, que le service de fabrication est dans le court terme et le service d'entretien, dans le long terme. 
Suivant l'horizon qu'on donne à l'activité de l'entreprise, on va arbitrer différemment. On retrouve là une question classique posée depuis Weber et Bourdieu sur le lien entre «psychologie économique » et horizon temporel des acteurs.

Ce que je viens de dire met en évidence une opposition très nette que j'ai par rapport à la tradition habermassienne qui considère la technique comme une "idéologie " qui pourrait se passer de toute valeur parce qu'elle serait sa propre valeur ${ }^{25}$. Or ce n'est pas du tout ce que je trouve dans une analyse concrète de la technique et du raisonnement technique. Les valeurs peuvent sembler obscurcies par le formalisme, mais, si on gratte un peu, on va effectivement les trouver. Ce qui m'intéresse, en sociologue, c'est de comprendre comment les gens sont amenés à raisonner en fonction du système de contraintes auxquelles ils sont confrontés. La métrologie n'est qu'un outil entre les mains des acteurs. Ce qui est intéressant, c'est de voir comment elle modifie les termes du débat, par sa façon de poser les valeurs. Mais elle n'éradique jamais les valeurs; c'est là une illusion technocratique partagée par les technocrates et leurs critiques.

Cela nous ramène à Imbert. Son étude du travail au sécateur est une belle fable sur la métrologie du travail ${ }^{26}$. Il avait été interpellé par les coupeuses des sarments de vigne qui se plaignaient des tarifs pratiqués. Il y avait deux tarifs suivant la taille du serment à couper. Elles considéraient que la différence d'effort physique n'était pas proportionnelle à la différence de tarifs: les gros serments étaient beaucoup plus durs à couper ; leur sur-tarif ne compensait pas la sur-fatigue qu'induisait leur coupe. Par une analyse fine de la mécanique de la main et du sécateur, Imbert montre qu'elles avaient raison. Pour autant, la solution adoptée ne sera pas de proportionner le tarif à l'effort mécanique, mais un nouveau tarif indifférencié plus élevé. Imbert, après avoir mené son travail d'analyse, n'est pas du tout choqué par cette résolution du conflit. Il ne considère pas que la mesure bio-énergétique du travail qu'il arrive à produire serait dans la capacité de résumer l'ensemble des valeurs qui se jouent dans cette situation professionnelle. Son expertise conduit à modifier d'une certaine manière le débat social qui se jouait entre les ouvrières et les employeurs, mais pas à fournir une réponse technocratique à la question. La mesure n'est pas inutile : elle montre que la sensation des ouvrières était fondée; pour autant, elle n'a pas à déboucher sur une rectification des tarifs proportionnant le salaire à l'effort mécanique.

II nous semble que dans votre œuvre, l'étude des sciences du travail peut se concevoir comme le moment fondateur d'une réflexion plus générale sur les conditions de possibilité d'une science de l'agir humain. Or, l'histoire des mesures du corps au travail montre, d'une part, la mobilisation pratique de ces mesures pour une « utilisation » plus « rationnelle » des corps et, d'autre part, l'incapacité de la mesure à saisir le caractère subjectif et historique de tout engagement corporel. Comment penser le rapport entre ces deux questions qui ouvre l'histoire des mesures du corps en activité ? Est-ce que cela signifie qu'aucune science de l'activité n'est possible puisque mesurer l'activité signifie reconduire le corps engagé du sujet à un modèle général de fonctionnement; ou au contraire, cela implique qu'il faut penser à une sorte de science du particulier, c'est-à-dire à une démarche capable de conserver ensemble la pérennité des procédés métrologiques et l'attention à la variabilité des expressions de l'activité?

Est-ce qu'on peut parler de science de l'activité ? Je n'en suis pas sûr. J'ai un peu de mal à répondre à cette question. J'ai le sentiment que le discours sur l'activité est un discours réactif par rapport à différents modèles de réification du travail. 
L'important est de rester vigilant par rapport à tout mode de réification du travail, mécanique comme socioéconomique (j'ai montré qu'elles peuvent être convergentes). À partir de là, est-ce qu'il faut installer une « science de l'activité »? Je n'en suis pas convaincu. Cela ne favoriserait-il pas une nouvelle forme de réductionnisme? Dans mon travail, la mesure est plutôt un objet d'observation qu'un instrument d'observation. Je considère que la mesure est une forme pratique de la vie individuelle comme sociale. Regarder la façon dont les gens mesurent, que ce soit d'une façon formelle ou informelle, dit quelque chose des valeurs qui les animent et de la façon dont ils s'orientent dans le monde. C'est en ce sens-là que j'ai proposé une sociologie de la mesure, non pas pour construire une mesure sociologique surplombante, mais, au contraire, pour prendre la mesure comme pratique dont l'extension est universelle ${ }^{27}$. Ce qui change suivant les sociétés et les contextes, c'est ce que l'on mesure et comment on mesure; ce n'est pas le fait de mesurer ou pas. C'est pourquoi la mesure est un analyseur de la vie sociale.

D'une façon générale, de mon point de vue, on peut toujours mesurer et les mesures ne sont jamais générales. Je m'oppose aux épistémologies qui distingueraient de façon radicale ce qui serait commensurable et ce qui ne le serait pas. On peut toujours construire des instruments de commensurabilité qui valent ce qu'ils valent et, de toute façon, la commensurabilité ne sera pas totale. Je veux dire, par là qu'on additionnera toujours des carottes et des navets d'une certaine façon. On prend deux grains de blé ; on estime que c'est la même chose et que l'on peut dire que l'on a « deux grains de blé ». Ce n'est vrai que jusqu'à un certain point. La sommation n'est valable que sous la supposition de l'identité de ces deux grains. Dès que l'on introduit d'autres caractéristiques (ils n'ont pas la même forme, le même poids, la même nature génétique, etc.), la sommation n'est plus possible. C'est pour cela que j'installe la mesure dans une pragmatique de l'action humaine.

Revenons à la question des sciences du travail. Est-ce que la bioénergétique fournit une mesure du travail ? Je ne le crois pas. On peut très bien élaborer une analyse énergétique du corps humain, mais cela ne nous permettra pas de distinguer ce qui, dans les dépenses énergétiques de l'homme, relève du travail ou n'en relève pas. C'est le paradoxe de Jules Amar dans sa position historique, au début du XXe siècle. Au moment où l'on a disposé effectivement d'un modèle bioénergétique cohérent pour penser la physiologie humaine, il avait perdu toute pertinence pour une science appliquée du travail. Je pense qu'on pourrait conclure de façon analogue d'instruments métrologiques plus récents appliqués au travail. Je connais mal les mesures de l'ergonomie contemporaine, mais je ne vois pas quelle mesure les ergonomes pourraient développer qui ne pourrait s'appliquer à une autre situation que le travail : si on s'intéresse à des problèmes de mouvement oculaire sur un écran, comment dissocier si vous appliquez la mesure de ce phénomène dans une situation de travail ou dans une situation de jeu sur ordinateur? La dimension proprement biomécanique ne nous dira jamais quelque chose sur le travail en tant que tel. Cela ne veut pas dire qu'on ne peut pas l'appliquer avec justesse au travail. C'est en ce sens-là que je ne crois pas à l'idée de science du travail. Je ne pense pas que ce soit la bonne manière de poser la question. En revanche, je pense qu'il y a des instruments de mesure pertinents qui peuvent s'appliquer à l'étude du travail. 


\section{NOTES}

1. VATIN François, «Les relations entre pensée technique et pensée économique », entretien avec Benjamin Ravier-Mazzocco ", e-Phaïstos, revue d'histoire des techniques, n² 2, 2013, p.86-89 2. BRAVERMAN Harry, Travail et capitalisme monopoliste, Paris, Maspero, 1976.

3. GALLE Raymond, VATIN François Vatin, Le modèle de fluidité. Étude économique et sociale d'une raffinerie de pétrole, Compte rendu d'une recherche financé par le C.O.R.D.E.S sur les transformations récentes de la division du travail dans les usines : étude d'une rafinerie de pétrole. Bandol, Laboratoire de Conjoncture et perspective.

4. TAYLOR Frederick Winslow, Organisation du travail et économie des entreprises, Paris, Éd. d'Organisation, 1990.

5. Voir par exemple, GEORGESCU-ROENGEN Nicholas, The Entropy Law and the Economic Process, s.l., Harvard University Press, 1971.

6. COULOMB Charles, "Résultats de plusieurs expériences destinées à déterminer la quantité d'action que les hommes peuvent fournir par leur travail journalier, suivant différentes manières dont ils emploient leurs forces ", Mémoire de l'Académie des sciences, 1799.

7. VATIN François, Le travail, sciences et société: essais d'épistémologie et de sociologie du travail, Bruxelles, Éd. de l'Université de Bruxelles, 1999.

8. CLOT Yves (dir.), Les histoires de la psychologie du travail : approche pluri-disciplinaire, Toulouse, Octares, 1996.

9. SARASIN Philipp, TANNER Jakob (dir.), Physiologie und industrielle Gesellschaft: Studien zur Verwissenschaftlichung des Körpers im 19. und 20. Jahrhundert, Deutsche Erstausgabe, Frankfurt am Main, Suhrkamp Verlag, 1998.

10. VATIN François, «L'homme mécanique et l'homme social. Une histoire des sciences du travail ", in CARROY Jacqueline, RICHARD Nathalie et VATIN François (dir.), L'homme des sciences de l'homme. Une histoire transdisciplinaire, Paris, Presses universitaires de l'Université Paris-Nanterre, 2013, p.65-85.

11. IMBERT Armand, «L'étude scientifique et expérimentale du travail professionnel. », L'année psychologique, 1906, vol.13, p.245-259.

12. AMAR Jules, Le moteur humain et les bases scientifiques du travail professionnel, Paris, H. Dunod et E. Pinat, 1914.

13. VATIN François, «Pierre-Édouard Lémontey, l'invention de la sociologie du travail et la question salariale ", Revue du MAUSS, 2006, t.27, nº1, p.398-420.

14. LÉMONTEY Pierre-Édouard, «Influence morale de la division du travail, considérée sous le rapport de la conservation du gouvernement et de la stabilité des institutions sociales. ", Revue du MAUSS, t.1, n²7, 2006 [1801].

15. MICHELET Jules, Le peuple, $5^{\mathrm{e}}$ édition Paris, Calmann Lévy, 1877.

16. FRIEDMANN Georges, La crise du progrès : esquisse d'histoire des idées, 1895-1935, Paris, Gallimard, 1936.

17. MARX Karl, Le capital, traduction de Joseph Roy, Paris, M. Lachâtre, 1872.

18. VATIN François, « Machinisme, marxisme, humanisme : Georges Friedmann avant et aprèsguerre ", Sociologie du travail, 2008, vol.46, n², p.205-223.

19. ROY Donald, "Quota Restriction and Goldbricking in a Machine Shop ", American Journal of Sociology, vol.57, $\mathrm{n}^{\circ} 5,1952$, p.427-442.

20. BELOT Émile, «Principes généraux de l'organisation systématique des machines et des usines", La Technique moderne, Octobre 1911; BELOT Émile, «Principes généraux de l'organisation systématique des machines et de l'industrie », La Technique moderne, avril 1918.

21. MÉDA Dominique, Le travail : une valeur en voie de disparition? Paris, Aubier, 1996. 
22. ARENDT Hannah, Condition de l'homme moderne, Paris, Calmann-Lévy, 1961.

23. WALLON Henri, Principes de psychologie appliquée, Paris, Armand Colin, 1930.

24. CROZIER Michel, Le Phénomène bureaucratique. Essai sur les tendances bureaucratiques des systèmes d'organisation modernes, Paris, Seuil, 1963.

25. HABERMAS Jürgen, La technique et la science comme « idéologie », Paris, Gallimard, (1968) 1973.

26. IMBERT Armand, «Étude expérimentale du travail professionnel ouvrier. Travail de coupage de sarments par boutures ", Revue d'économie politique, Janvier 1909. Voir LE BIANIC Thomas, VATIN François, « Armand Imbert (1850-1922), la science du travail et la paix sociale », Travail et Emploi, $\mathrm{n}^{\circ} 111$, juillet-septembre 2007, p.7-19.

27. VATIN François (dir.), Évaluer et valoriser. Une sociologie économique de la mesure, Toulouse, Presses universitaires du Mirail, 2009 et 2013.

\section{INDEX}

Mots-clés : histoire des techniques, sociologie de l'activité, sociologie du travail

Keywords : history of technology, sociology of activity, sociology of work

\section{AUTEURS}

\section{BARTHÉLÉMY DURRIVE}

Agrégé de philosophie, docteur en philosophie et histoire des sciences, Barthélemy Durrive enseigne actuellement la philosophie dans le secondaire. Après une thèse consacrée à l'histoire de la constitution du programme de recherches intitulé « physiologie du travail » en France au tournant du XXe siècle, ses recherches portent actuellement sur l'histoire et l'épistémologie de l'ergonomie.

\section{MARCO SARACENO}

Docteur en Sociologie et Histoire des Sciences, Marco Saraceno est actuellement chercheur postdoctorat au CETCOPRA, Université Paris 1 Panthéon-Sorbonne. Il s'intéresse à l'histoire de la psychophysiologie de l'activité motrice avec une attention particulière à l'évolution de ses techniques de mesure et à sa relation avec les autres sciences humaines. Dans ce contexte, il a étudié l'histoire de la notion de fatigue en observant le rôle qu'elle joue durant le « moment 1900 » dans la « fondation » des sciences sociales et dans la rhétorique décadentiste de l'époque. Il travaille actuellement sur la socio-histoire des capteurs de l'activité physique et sur l'évolution de leurs techniques d'enregistrement graphique. 